\title{
Donor human milk in Neonatal Intensive Care Unit - to whom, how much and how long?
}

\author{
Izabela M. Lehman, Barbara Broers, Matylda Czosnykowska-Lukacka, \\ Weronika Wesolowska, Lucyna Swiderska, Barbara Krolak-Olejnik \\ Departament of Neonatology, Wroclaw Medical University, Poland
}

\begin{abstract}
Objectives: The aim of the study was to present the variability of patients who received donor human milk (DHM) during Neonatal Intensive Care Unit (NICU) hospitalization, including time of its usage and volume of portions.

Material and methods: A retrospective analysis of data was conducted for all infants admitted to the NICU at the University Hospital during the first year of the Human Milk Bank operation. One-way analysis of variance in the intergroup scheme, Kruskal-Wallis variance analysis with the Jonckheere-Tepstra test, correlation analysis using Pearson's $r$ and Spearmann's rho, frequency analysis using the Fisher's exact test were used to conduct analyses.

Results: 133 newborns received DHM. 3 groups of neonates were identified: < 32 0/7 weeks, 32 0/7-36 6/7 weeks and $>37$ 0/7 weeks of gestational age (GA). Time of DHM supplementation was similar in all groups and does not differ depending on the GA but preterm infants received the smallest total volume of DHM. However, infants $>37$ weeks of GA had almost a threefold greater chance of abandoning breastfeeding than the others (odds ratio $(\mathrm{OR})=2.89,95 \% \mathrm{Cl}$ : 0.69-12.20). There was a statistically significant, weak negative correlation between period of total parenteral nutrition and the volume of milk from the bank: rho $=-0.194 ; p=0.026$.

Conclusions: The DHM supply did not have a negative impact on lactation and breastfeeding. Stimulation of lactation was necessary for 5-7 days. The time of DHM supply was the same regardless of GA. The majority of infants were breastfed or received only MOM on the day of discharge from the hospital.
\end{abstract}

Key words: human milk bank; neonatal intensive care unit; neonate; preterm infant; breastfeeding

Ginekologia Polska 2019; 90, 9: 534-538

\section{INTRODUCTION}

Enteral feeding of newborns treated in a Neonatal Intensive Care Unit (NICU) is a challenge for medical staff. World Health Organization (WHO) [1, 2], American Academy of Pediatrics (AAP) [3] and The European Society for Pediatric Gastroenterology, Hepatology and Nutrition (ESPGHAN) [4] have recognized the superiority of mother's own milk (MOM) over all infant formulas. It is not always possible to get milk from biological mother just after delivery, then the second choice is the donor human milk (DHM). This is compatible with the international guidelines [1-4], which states that: "Donor human milk from a professional milk bank is the second best choice for newborns after mother's own milk."

The main recipients of DHM are premature newborns. In the last decade, the use of pasteurized DHM has become the standard of care for very low birthweight $\left(\mathrm{VLBW}_{;}<1500 \mathrm{~g}\right)$ infants when MOM is not available [5, 6]. The usage of
DHM by other high-risk newborns hospitalized in NICU also increases, primarily for full-term infants with severe complications and for infants in pre- and postoperative period [7]. Human milk is an ideal food for newborns, both as a full-fledged food and as a missing link in the immune protection of the baby. The activity of Human Milk Banks creates equal opportunities for all infants to access the best nourishment.

\section{OBJECTIVES}

The aim of the study was to present the variability of patients who received DHM during hospitalization, including time of its usage and volume of portions. The study also concerned the duration time of total parenteral nutrition and the moment of hospitalization when the infants had been fed only with MOM, and the method of feeding at the time of discharging from the hospital. 


\section{MATERIAL AND METHODS}

A retrospective analysis of available data was conducted for all infants admitted to the NICU of Department of Neonatology at University Hospital, during the first year of Human Milk Bank (HMB) activity between 10/02/2017 and $31 / 12 / 2017$. The study received ethical approval from the Ethics Committee (177/2015 and 242/2017). Permission to apply DHM had to be given by the infants' legal guardian.

\section{Feeding policy}

Minimal enteral feeding with MOM or DHM was introduced, usually within the first 6 hours of life. Mothers were also encouraged to obtain colostrum by initiation of lactation during the first 6 hours post-delivery. After few hours of priming the infant's intestine, feeding was increased by $15-30 \mathrm{~mL} / \mathrm{kg} /$ day depending on the maturity and feeding tolerance of the infant. When enteral feeding has reached $100 \mathrm{~mL} / \mathrm{kg} /$ day, parenteral nutrition was discounting.

\section{Preparation of DHM}

HMB in Department of Neonatology in the University Hospital is the seventh bank operating in Poland, associated in the European Milk Bank Association (EMBA). Donors must undergo rigorous screening before donation, concerning the health condition, blood and milk analysis. The milk is subject to Holder Pasteurization $\left(62.5^{\circ} \mathrm{C}\right.$ for 30 minutes) and then is frozen, while awaiting for final results of microbiological cultures.

\section{Infant formula}

If after 14 days of using DHM, MOM was not in sufficient quantities to cover the nutritional needs, neonates were given infant formula according to the gestational age (GA).

\section{Data collection}

All data was collected from paper and electronic medical database. Maternal data regarding age, pregnancy, delivery and health were analyzed. Neonatal data concerning the gestational age, birth weight, clinical condition of newborns, including the need for intensive therapy, time of total parenteral nutrition (TPN) supply, DHM volume given to the child and time of breast milk supply were analyzed. The final point of the analysis was the method of feeding the child at discharge from the NICU.

\section{Statistics}

Two sets of analyzes were carried out. At first, descriptive characteristics of children and mothers participating in the study were reported. The Kołmogorow-Smirnov test was used to determine if the sample belongs to population with a normal deviation. The Mann-Whitney U test's purpose was to compare continuous data to avoid assuming a normal distribution. Descriptive statistics were used to demon- strate the mean \pm standard deviation or median (min-max) for constant variables, whereas nominal variables were expressed as case number and percentages. One-way analysis of variance in the intergroup scheme, Kruskal-Wallis variance analysis with the Jonckheere-Tepstra test, correlation analysis using Pearson's $r$ and Spearmann's rho, frequency analysis using the Fisher's exact test were used to conduct analyses. All statistical analysis were performed using IBM SPSS Statistics 23. A p value $<0.05$ was considered statistically significant in all analyses.

\section{RESULTS}

There were 2560 newborns hospitalized in the Neonatology Department, including 248 neonates admitted to NICU between 10/02/2017 and 31/12/2017. During the first year of Human Milk Bank activity 133 newborns had been receiving DHM. Each newborn had been receiving colostrum and MOM, but nutrition was supplemented with DHM to quickly terminate TPN. The characteristics of newborns and their mothers are presented in the Table 1.

\section{Donor human milk and gestational age of the newborn infants}

A hypothesis was verified concerning the relationship between milk supply from the HMB and gestational age (GA). For this purpose, a group of children included in the study was divided into three subgroups depending on GA: under 32 0/7, between 32 0/7 and 36 6/7, over 37 0/7 weeks. In such separate groups two factors were compared: volume of DHM portion $(\mathrm{mL})$ and number of days when DHM was provided to newborns. There were substantial differences between the groups: $[H(2)=17, p<0.001, \eta 2=0.13]$. In addition a statistically significant trend was captured: the higher the gestational age, the higher DHM supply $(J=2910.00$, $p=0.002)$. Results are summarized in Figure 1.

Days of using DHM was analyzed, a one-way analysis of variance was performed in 3 groups of neonates. The result was not statistically significant: $F(2 ; 130)=2.58$; $p=0.079$. The value of coefficient $\omega 2$ was 0.02 . Days of supplied of DHM was similar in all groups and does not differ depending on the GA of newborn infants. The results are presented in the Table 2 .

\section{DHM and duration of total parenteral nutrition (TPN)}

Duration of TPN related with the supply of DHM in terms of its quantity $(\mathrm{mL})$ and period of time were analyzed. To this end, a correlation analysis was carried out with the Spearman rho coefficient comparing the above-mentioned variables with period of time of TPN (in days).

There was a statistically significant, weak negative correlation between days of TPN and the volume of milk from 
Table 1. Demographics of newborns who received DHM and their mothers

\begin{tabular}{|c|c|}
\hline \multicolumn{2}{|l|}{ Maternal characteristics $(n=103)$} \\
\hline Age [years] $\mathrm{M} \pm \mathrm{SD}$, (min-max) & $32.42 \pm 5.12(18-45)$ \\
\hline \multicolumn{2}{|l|}{ Pregnancy complications, $\mathbf{n}(\%)$} \\
\hline Gestational diabetes & $15(14.6)$ \\
\hline Preeclampsia & $20(19.4)$ \\
\hline Twin gestation & $18(17.5)$ \\
\hline Triplet gestation & $3(2.9)$ \\
\hline Vaginal delivery, n (\%) & $17(16.5)$ \\
\hline Cesarean section, $\mathrm{n}(\%)$ & $86(83.5)$ \\
\hline \multicolumn{2}{|l|}{ Infant characteristics ( $n=133$ ) } \\
\hline Male n (\%) & $77(58)$ \\
\hline Female n (\%) & $56(42)$ \\
\hline Gestational Age [weeks] M \pm SD, (min-max) & $33.98 \pm 3.4(23-41)$ \\
\hline Birth weight $[\mathrm{g}] \mathrm{M} \pm \mathrm{SD}$, (min-max) & $\begin{array}{l}2108.95 \pm 737.62 \\
(510-4090)\end{array}$ \\
\hline Apgar 1 min. Me (min-max) & $8(0-10)$ \\
\hline Apgar 5 min. Me (min-max) & $8(4-10)$ \\
\hline Adaptive disorders & $32(24 \%)$ \\
\hline Respiratory disorders & $84(63 \%)$ \\
\hline Hipotermia & $3(2.6 \%)$ \\
\hline Triplets & $9(6.8 \%)$ \\
\hline Congenital malformations & $5(3.8 \%)$ \\
\hline Post-surgery treatment & $4(3 \%)$ \\
\hline Days of Hospitalization $\mathrm{M} \pm \mathrm{SD}$, (min-max) & $22.84 \pm 15.72(5-90)$ \\
\hline Weight at discharge $[\mathrm{g}] \mathrm{M} \pm \mathrm{SD}$ & $2513.7 \pm 510.9$ \\
\hline \multicolumn{2}{|l|}{ Donor Human Milk } \\
\hline Days $M \pm S D$, (min-max) & $6.09 \pm 4.98(1-23)$ \\
\hline Volume $\mathrm{mL} M \pm S D$, (min-max) & $\begin{array}{l}703.33 \pm 974.35 \\
(3-4726)\end{array}$ \\
\hline
\end{tabular}

$\mathrm{M}$ - medium; $\mathrm{Me}$ - median; SD — standard deviation; min and max — the lowest and highest value of the distribution

$$
1500
$$

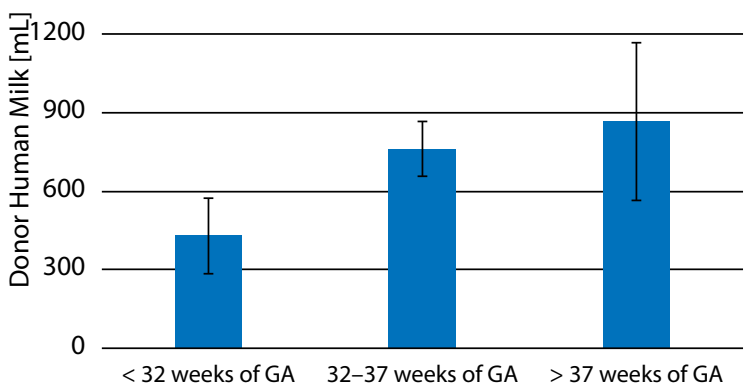

Figure 1. Volume of DHM and gestational age of infants

HMB: rho $=-0.194 ; p=0.026$. The longer TPN period, the smaller DHM supply. However, in case of comparing the days
Table 2. Comparison of days of DHM supply in the groups of the newborn infants

\begin{tabular}{|l|l|l|l|l|l|}
\hline \multicolumn{1}{|c|}{ Week of GA } & $\mathbf{n}$ & $\mathbf{M}$ & $\mathbf{S E}$ & LL & UL \\
\hline$<320 / 7$ & 29 & 5.03 & 0.96 & 3.06 & 7.01 \\
\hline $320 / 7-366 / 7$ & 87 & 6.78 & 0.54 & 5.70 & 7.86 \\
\hline$\geq 370 / 7$ & 17 & 4.35 & 0.81 & 2.63 & 6.08 \\
\hline
\end{tabular}

$\mathrm{n}$ - number of observations; $\mathrm{M}$ - medium; $\mathrm{SE}$ - standard error; $95 \% \mathrm{Cl}$

- confidence interval for the difference between means; $\mathrm{LL}$ and $\mathrm{UL}$ - the

lower and upper limits of the confidence interval of milk with BM

of DHM supply and the days of TPN, the relationship did not turn out to be statistically significant: $r h o=-0.061 ; p=0.489$.

\section{DHM and exclusively feeding with mother's own milk on the day of discharge from the NICU}

Relative risk (RR) of non-breastfeeding for infants born > 37 weeks of GA compared with the rest of newborns were calculated. Frequency analysis using the exact Fisher test showed a statistically insignificant result: $p=0.149$. The vast majority of infants received either exclusively MOM or mixed feeding at discharge, both in the group $>37$ weeks of GA (82.4\%) and in preterm infants (93.1\%). The calculated value of the odds ratio (OR) indicates that newborn infants > 37 weeks of GA have almost a threefold greater chance of abandoning breast-feeding than the others $(\mathrm{OR}=2.89,95 \% \mathrm{Cl}: 0.69-12.20)$. Subsequently, the odds ratio (OR) was calculated for exclusive mother's milk feeding the neonates born < 32 weeks of GA compared with other infants. Frequency analysis using the exact Fisher test showed a statistically insignificant result: $p=0.475$. The majority of infants received only MOM at discharge, both in the group $<32$ weeks of GA (69.0\%) and in other infants (76.0\%). The calculated value of OR indicates that infants born > 32 weeks of GA have a slightly higher chance of feeding exclusively with MOM than the others ( $O R=1.42$, $95 \% \mathrm{Cl}: 0.58-3.52)$. On the other hand, the relative risk of exclusively breastfeeding is similar in both groups ( $R R=0.91$, 95\% Cl: 0.70-1.19). However, since the Fisher test result did not turn out to be statistically significant, and the $95 \%$ confidence interval reported for the odds ratio and relative risk contains the value 1 , it should be considered that the chance of breastfeeding alone at discharge is not meaningfully greater for any group. The results are summarized in Table 3.

\section{DISCUSSION}

Numerous recommendations confirm, that MOM is always preferred, but DHM should be used for high-risk infants, when the mother's milk is not available $[3,4,7,8]$. $\mathrm{DHM}$ does not replace MOM but is a supplement. Increas- 


\begin{tabular}{|c|c|c|c|c|}
\hline \multirow{2}{*}{$\begin{array}{l}\text { Exclusively } \\
\text { MOM at the day } \\
\text { of discharge }\end{array}$} & & \multicolumn{2}{|l|}{ GA } & \multirow{6}{*}{$\begin{array}{l}p=0.475 \\
O R=1.42\end{array}$} \\
\hline & & $\begin{array}{l}<32 \text { weeks } \\
\text { of GA }\end{array}$ & $\begin{array}{l}>32 \text { weeks } \\
\text { of GA }\end{array}$ & \\
\hline \multirow{2}{*}{ No } & $N$ & 9 & 25 & \\
\hline & $\%$ & $31.00 \%$ & $24.00 \%$ & \\
\hline \multirow{2}{*}{ Yes } & $N$ & 20 & 79 & \\
\hline & $\%$ & $69.00 \%$ & $76.00 \%$ & \\
\hline
\end{tabular}

ingly, the multiple beneficial outcomes attributed only to MOM can be generalized to DHM [9].

There are no explicit recommendations regarding the supply of DHM in NICU. Indications for administering milk from the bank are usually: prematurity $(<28,<34,<36$ weeks of $G A)$, very low birth weight infant (VLBW), extremely low (ELBW) and low birth weight (LBW). Fewer beneficiaries of DHM are infants who require intensive treatment or newborns in the pre- and postoperative period. In Poland the order is based on the recommendations of the Human Milk Banking Association of North America (HMBANA) [10]. Priority should be given to provide DHM to infants $<1500 \mathrm{~g}$ birth weight for minimal enteral feeding, as quickly as the patient's condition allows [11].

According to the recommendations in force in Poland, the minimum enteral feeding was started from the mother's colostrum during first 6 hours after birth and if possible even in the first 2 hours of life. Newborn infants received DHM in the absence of MOM. When enteral feeding has reached $100 \mathrm{~mL} / \mathrm{kg} /$ day, parenteral nutrition discounted. Children were receiving DHM until 14 days of life, especially near term infants, because longer usage caused mother's negative motivation to stimulate and maintain lactation.

Research on the clinical benefits of using DHM in NICU is underway. The influence of human milk on the health-promoting effects is best documented $[9,12,13]$. The early nutrition plays an important role on later cognition [14]. The duration of TPN in all analyzed groups was similar. It did not differ significantly. Also, the duration of feeding with DHM did not differ between three groups of infants divided according to the gestational age. Although no statistical significance was obtained, infants born between 32 0/7 and 36 6/7weeks of $\mathrm{GA}$ required DHM for the longest time. Mothers of these newborns required special lactation care, and the stimulation of lactation was the most difficult. At the same time, the preterm required the shortest treatment in NICU, because they had mainly respiratory distress syndrome, transient tachypnea of the newborn or adaptive disorders in the perinatal period. Infants received DHM to replenish the volume of MOM and no donor milk was used instead of mother's milk.

However, the nutritional value of DHM compared to mother's raw milk needs further research. Low weight gain, body length, and head circumference in the early postnatal period may be associated with the preparation of milk. DHM undergoes pasteurization which influences its bioactive properties. However, donor milk maintains documented advantages compared to formula $[9,15]$.

Donor human milk usually comes from women after stabilization of lactation, when the protein content in breast milk is reduced. The premature milk composition differs significantly from the mature milk $[16,17]$. The system of DHM energy content categorization and distribution would improve energy intake from human milk [18].

Fewer data are available regarding the use of DHM in other high-risk infants, including infants with abdominal wall defects, such as gastroschisis or omphalocele, and other conditions, such as perinatal asphyxia [11]. Nonetheless, some infants with these conditions or other neonatal disorders may benefit from DHM either, because of a direct effect on intestinal growth or improved feeding tolerance [19]. Our retrospective observations have shown that children always received colostrum and raw mother's milk, and DHM was used to rapidly increase enteral nutrition, supplementing but not replacing MOM.

Patients in the NICU in University Hospital were not only premature neonates and newborns with symptoms of respiratory failure, but also infants treated with hypothermia after perinatal asphyxia, infants after surgical intervention due to esophagus atresia, intestinal obstruction, overgrown anus/rectus or gastroschisis. Newborns received DHM during the initial stages of maternal lactation when the volume of milk was insufficient, while it was possible to increase the volume of enteral nutrition and rapid withdrawal of TPN. Among our patients, no complications related to the rapid increase of enteral nutrition were observed, no necrotizing enterocolitis cases were also recognized.

A strategy aiming to promote and support breastfeeding should be carried out in every maternity or children's hospital where premature infants are born or treated and cared for after birth. Early intervention with milk expression after delivery (ideally within 6 hours) is critical for milk production; therefore, mothers should be educated a method of milk expression within this time frame. Presence of an HMB does not compete with breastfeeding, but decreases the utilization of formula in a NICU and increases exclusive breastfeeding rates at discharge [20-22]. In our analyses the vast majority of infants received either exclusive or partial MOM at discharge, both in the group of gestational age $>37$ weeks (82.4\%) and in premature infants (93.1\%).

\section{CONCLUSIONS}

Neonates born before 32 0/7 week of GA, as expected, received the smallest volume of $\mathrm{DHM}$, but, the time of supply was almost the same irrespective of GA. The initiation and 
maintenance phase of lactation of newborn infants' mothers allowed to obtain the sufficient volume of MOM in 5-7 days after delivery. Despite many scientific studies and published clinical trials, it has not been clearly established and there are no recommendations regarding the supply of DHM in $\mathrm{NICU}$, i.e. the time of supply, volume and type of patients who should receive breast milk. However, replenishing the MOM and the initiation and maintenance of lactation allowed exclusively breastfeeding or feeding only with MOM for all newborns. Further studies on the short-and long-term effects of DHM on child development are needed, as well as the guidelines for the use of DHM in the NICU.

Based on the obtained data, we recommend supplying DHM to all newborns treated in NICU when mother's milk is not available or if its volume is too small to fulfill the infants' nutritional needs. However, it is to be remembered that DHM should be used as a supplement to breast milk and not instead of MOM. Further research is needed to help building universally accepted recommendations.

\section{Acknowledgments}

The study was approved by Bioethical Committee at the Medical University (177/2015 and 242/2017). Permission to apply DHM had to be given by the child's legal guardian. Thanks to Brian Hughes for proofreading.

\section{REFERENCES}

1. WHO/UNICEF meeting on infant and young child feeding. Journal of Nurse-Midwifery. 1980;25(3):31-38, doi: 10.1016/0091-2182(80)90051-8.

2. WHO \& UNICEF. Global strategy for infant and young child feeding. Geneva, 2003.

3. American Academy of Pediatrics. Breastfeeding and the use of human milk. 2012; 29(3): 827-841.

4. Arslanoglu S, Corpeleijn W, Moro G, et al. ESPGHAN Committee on Nutrition. Donor human milk for preterm infants: current evidence and research directions. J Pediatr Gastroenterol Nutr. 2013; 57(4): 535-542, doi: 10.1097/MPG.0b013e3182a3af0a, indexed in Pubmed: 24084373.

5. Moro GE, Arslanoglu S, Bertino E, et al. American Academy of Pediatrics, European Society for Pediatric Gastroenterology, Hepatology, and Nutrition. XII. Human Milk in Feeding Premature Infants: Consensus Statement. J Pediatr Gastroenterol Nutr. 2015; 61 Suppl 1: S16-S19, doi: 10.1097/01.mpg.0000471460.08792.4d, indexed in Pubmed: 26295999.
6. Perrine CG, Scanlon KS. Prevalence of use of human milk in US advanced care neonatal units. Pediatrics. 2013; 131(6): 1066-1071, doi: 10.1542/peds.2012-3823, indexed in Pubmed: 23669517.

7. Arslanoglu S, Corpeleijn W, Moro G, et al. ESPGHAN Committee on Nutrition. Donor human milk for preterm infants: current evidence and research directions. J Pediatr Gastroenterol Nutr. 2013; 57(4): 535-542, doi: 10.1097/MPG.0b013e3182a3af0a, indexed in Pubmed: 24084373.

8. WHO. Guidelines on Optimal Feeding of Low Birthweight Infants in Low- and Middle-Income Countries. Geneva, 2011.

9. Meier P, Patel A, Esquerra-Zwiers A. Donor Human Milk Update: Evidence, Mechanisms, and Priorities for Research and Practice. J Pediatr. 2017; 180: 15-21, doi: 10.1016/j.jpeds.2016.09.027, indexed in Pubmed: 27773337.

10. Human Milk Banking Association of North America. https://www. hmbana.org/ (15.05.2018).

11. COMMITTEEON NUTRITION, SECTIONON BREASTFEEDING, COMMITTEEON FETUS AND NEWBORN. Donor Human Milk for the High-Risk Infant: Preparation, Safety, and Usage Options in the United States. Pediatrics. 2017; 139(1), doi: 10.1542/peds.2016-3440, indexed in Pubmed: 27994111.

12. Cristofalo EA, Schanler RJ, Blanco $C L$, et al. Randomized trial of exclusive human milk versus preterm formula diets in extremely premature infants. J Pediatr. 2013; 163(6): 1592-1595.e1, doi: 10.1016/j. jpeds.2013.07.011, indexed in Pubmed: 23968744.

13. Corpeleijn WE, de Waard M, Christmann V, et al. Effect of Donor Milk on Severe Infections and Mortality in Very Low-Birth-Weight Infants:The Early Nutrition Study Randomized Clinical Trial. JAMA Pediatr. 2016; 170(7):654661, doi: 10.1001/jamapediatrics.2016.0183, indexed in Pubmed: 27135598.

14. Embleton ND. Early nutrition and later outcomes in preterm infants. World Rev Nutr Diet. 2013; 106: 26-32.

15. Meier PP, Johnson TJ, Patel AL, et al. Evidence-Based Methods That Promote Human Milk Feeding of Preterm Infants: An Expert Review. Clin Perinatol. 2017; 44(1): 1-22, doi: 10.1016/j.clp.2016.11.005, indexed in Pubmed: 28159199.

16. Boyce $\mathrm{C}$, Watson $\mathrm{M}$, Lazidis $\mathrm{G}$, et al. Preterm human milk composition: a systematic literature review. Br J Nutr. 2016; 116(6): 1033-1045, doi: 10.1017/S0007114516003007, indexed in Pubmed: 27522863.

17. Castellote C, Casillas R, Ramírez-Santana C, et al. Premature delivery influences the immunological composition of colostrum and transitional and mature human milk. J Nutr. 2011; 141(6): 1181-1187, doi: 10.3945/jn.110.133652, indexed in Pubmed: 21508211.

18. Simpson JH, McKerracher L, Cooper A, et al. Optimal Distribution and Utilization of Donated Human Breast Milk. J Hum Lact. 2016; 32(4): 730 734, doi: 10.1177/0890334416653738, indexed in Pubmed: 27364932.

19. Kohler JA, Perkins AM, BassWT. Human milk versus formula after gastroschisis repair: effects on time to full feeds and time to discharge. J Perinatol. 2013; 33(8): 627-630, doi: 10.1038/jp.2013.27, indexed in Pubmed: 23519369.

20. Arslanoglu $S$, Moro GE, Bellù $R$, et al. Presence of human milk bank is associated with elevated rate of exclusive breastfeeding in VLBW infants. J Perinat Med. 2013;41(2): 129-131, doi: 10.1515/jpm-2012-0196, indexed in Pubmed: 23241582.

21. de Halleux V, Pieltain C, SenterreT, et al. Use of donor milk in the neonatal intensive care unit. Semin Fetal Neonatal Med. 2017; 22(1): 23-29, doi: 10.1016/j.siny.2016.08.003, indexed in Pubmed: 27649995.

22. Geddes D, Perrella S. Breastfeeding and Human Lactation. Nutrients. 2019; 11(4), doi: 10.3390/nu11040802, indexed in Pubmed: 30970568 